\title{
Evaluation of the partitioned mechanical properties and the hall-petch relationship of cast aluminum alloy cylinder head
}

\author{
Kangjie Yan ${ }^{1}$, Weiqing Huang ${ }^{1 *}$, Zhengxing Zuo ${ }^{1}$, Jinxiang Liu ${ }^{1}$, Peirong Ren ${ }^{1}$, Dingyun \\ $\mathrm{Hu}^{2}$, and Chengzhang Zhao ${ }^{1}$ \\ ${ }^{1}$ School of Mechanical Engineering, Beijing Institute of Technology, Beijing, China \\ ${ }^{2}$ China North Engine Research Institute(Tianjin), Tianjin, China
}

\begin{abstract}
In view of the non-uniform distribution of mechanical properties of cast aluminum alloy cylinder head, the mechanical properties evaluation and microstructure heterogeneity of cylinder head were studied. The results showed that the head plate position of the cylinder head has the best mechanical properties and microstructure characterization, followed by the floor plate and the thick partition plate. The mechanical properties of the floor plate position attenuate with increasing temperature. From $23^{\circ} \mathrm{C}$ to $300^{\circ} \mathrm{C}$, the tensile strength and yield strength decrease in the same range, but the break elongation changes most obviously. The mechanical properties and microstructure characterization of cylinder head in-situ sampling satisfy the Hall-Petch relationship. If the required ultimate tensile strength is not less than $255 \mathrm{MPa}$, the upper threshold of the grain size, by considering the error limit of the Hall-Petch relationship, is $603.4 \mu \mathrm{m}$, and the upper threshold of secondary dendrite arm spacing is $69.1 \mu \mathrm{m}$. Meanwhile, established the relationship between hardness and yield strength, the average error of the nonlinear model is $4.35 \%$. The prediction accuracy of the nonlinear model is sufficient to meet the actual needs of the engineering.
\end{abstract}

\section{Introduction}

Due to the advantages of high explosion pressure, high power output and high combustion efficiency, diesel engine occupies an absolute share in the heavy-duty civilian and military vehicle market. The state strictly limits the emissions of environmental pollutants, requiring heavy-duty diesel vehicles to reduce nitrogen oxide and particulate emissions by more than $60 \%$, In order to meet increasingly stringent emission regulations, it is necessary to continue to improve the combustion efficiency and power density of diesel engines, which undoubtedly worsens its operating conditions. While rising performance, reliability and strength of the key components of diesel engine also encountered unprecedented challenges. As one of the most complex components in internal combustion engines, the cylinder head is not only arranged with intake and exhaust passages, cooling water chambers, fuel injection seat hole and other structures, but also forms the main combustion chamber with the piston

* Corresponding author: hwq@, bit.edu.cn 
crown, cylinder head gasket and cylinder liner. Besides the complexity of structure, the cylinder head is subjected to various thermal-mechanical loads simultaneously during the working process, the complexity of the structure and the diversity of the bearing capacity make the cylinder head one of the most vulnerable parts of the power system.

Under the current technological level, the casting process is a necessary link for the mass production of cylinder heads. Due to the advantages of good casting performance, low density, high strength, and corrosion resistance, aluminum-silicon alloy has become a lightweight material for casting that is widely used in automotive, aviation, shipbuilding and other industrial fields. Highly-intensified diesel engines also widely use aluminum alloy as the material of the body and cylinder head to achieve the purpose of lightweight ${ }^{1}$. However, when the casting process is applied to the mass production process, the problem of spatial dispersion in microstructure caused by the complexity of casting process and structure cannot be avoided. Because of the large wall thickness step, local structure size mutation and high curvature of the surface in the key parts of the cylinder head, the solidification rate of liquid phase is different at different positions, accompanied by local eddy current phenomenon, which leads to obvious differences in secondary dendrite arm spacing (SDAS), grain size and eutectic silicon morphology at different positions. The inhomogeneity of mechanical properties caused by microstructure heterogeneity becomes an important factor restricting the further improvement of cylinder head performance and the overall strength evaluation and reliability problems of cylinder heads under non-uniform mechanical properties need to be resolved urgently. In addition, the floor plate area of the cylinder head is under the action of high temperature and high pressure for a long time, the aluminum-silicon alloy strengthened precipitates are continuously coarsened, the mechanical properties are constantly attenuated. The thermal instability of the material greatly limits the further improvement of the structural product performance ${ }^{2,3}$.

Many scholars at home and abroad have carried out partition evaluation on the mechanical properties of complex castings, The results show that the microstructure of each position of the casting is not the same, the mechanical properties of the casting are non-uniformly distributed with space, there are differences between the results of in-situ sampling experiment and ingot sampling experiment, so the mechanical properties of complex castings cannot be homogenized ${ }^{4-7}$. Meanwhile, other studies also show that the microstructure SDAS and grain size have a great influence on the mechanical properties of the materials. The grain refinement and smaller SDAS of Al-Si alloy increase the number of grain boundaries which can inhibit the slippage of dislocations and finally improve the strength and ductility of the material. The effect of grain size and SDAS on yield strength can be expressed by Hall-Petch relationship ${ }^{8-11}$. The morphology, size and distribution of eutectic silicon will also influence the mechanical properties of the material. The coarser acicular eutectic silicon is likely to cause stress concentration, which will adversely affect the mechanical properties of the material $^{12-14}$.

At present, there are few researches on the evaluation of the partitioned mechanical properties and the relationship between microstructure and mechanical properties of in-situ sampling of cylinder heads. The aim of this work is therefore to carry out experiments to evaluate the mechanical properties and microstructure of cylinder heads in different positions. Study the Hall-Petch relationship between the microstructure parameters and mechanical properties of in-situ sampling of cylinder heads, and establish the relationship between hardness and yield strength. The allowable thresholds of SDAS and grain size were calibrated based on Hall-Petch relationship, in order to guide the improvement of casting process in the future. 


\section{Experimental}

\subsection{Sampling scheme}

Taking the cooling rate as the sampling reference standard, in-situ samplings of cast aluminum alloy cylinder heads were carried out. The cooling rate of the head plate is the fastest due to the arrangement of the chill, while the thick partition plate is poor in heat dissipation and the cooling rate is the slowest, and take floor plate into account which bears high temperature and high pressure. The order of cooling rate is head plate, floor plate and thick partition plate. The size of tensile specimens is designed according to GB/T228.1-2010 and GB/T 228.2-2015 standards. Fig. 1 and Fig.2 are the dimensions of tensile specimens and the distribution of sampling locations, respectively. The chemical composition of the aluminum alloy cylinder head is shown in Table 1.

Table1 Chemical composition(wt.\%) of Al-Si alloy.

\begin{tabular}{|c|c|c|c|c|c|c|}
\hline $\mathbf{S i}$ & $\mathbf{M g}$ & $\mathbf{C u}$ & $\mathbf{M n}$ & $\mathbf{T i}$ & $\mathbf{S r}$ & $\mathbf{A l}$ \\
\hline 7 & 0.26 & 1.5 & 0.15 & 0.1 & 0.02 & Bal. \\
\hline
\end{tabular}

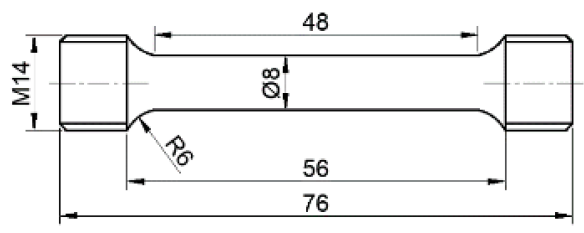

Fig. 1. Dimensions of the tensile specimens.

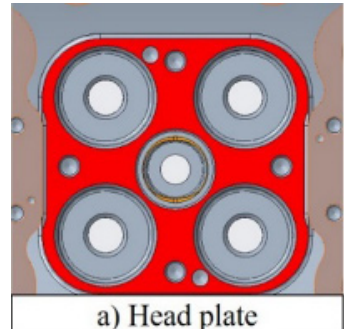

a) Head plate

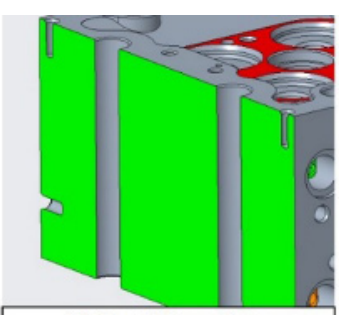

b) Partition plate

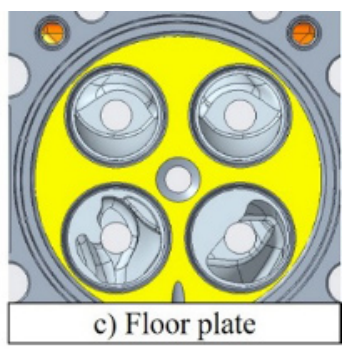

c) Floor plate

Fig. 2. Schematic diagram of sampling location.

\subsection{Mechanical property tests}

Considering that the floor plate is in the working environment of high temperature and high pressure, high temperature mechanical properties should be studied. The tensile tests at room temperature and high temperature were carried out on the MTS809 electric servo pull torsion composite test machine based on GB/T 228.1-2010 and GB/T 228.2-2015. The strain rate is $0.00025 \mathrm{~s}^{-1}$ at room temperature and $0.0002 \mathrm{~s}^{-1}$ at high temperature. After completing the tensile test, the hardness was tested on the corresponding sample by $320 \mathrm{HBS}-3000$ Brinell hardness tester, according to GB/T 231.1-2018.

\subsection{Quantification of microstructure}

Measure the microstructure parameters of the samples which has completed the mechanical 
properties experiment. After grinding and polishing, the metallographic samples were corroded in alcohol solution containing $0.5 \%$ nitric acid. The microstructure of the samples was observed under Zeiss Zim optical microscope. The microstructure parameters were quantitatively measured by Image Pro Plus software. The parameters include grain size, SDAS, and aspect ratio of eutectic silicon. SDAS is measured by taking the average value $\mathrm{SDAS}=\mathrm{L} / \mathrm{N}$ for multiple secondary cell spacing, where $\mathrm{L}$ is the total length between two edges of the continuous arranged cell, and $\mathrm{N}$ is the number of the measured cell. Aspect ratio is the ratio of major axis to minor axis of eutectic silicon equivalent ellipse.

\section{Results and discussion}

\subsection{Mechanical properties}

The partitioned mechanical properties of aluminum alloy cylinder heads are shown in Table 2. Fig. 3 shows the ratio of the mechanical properties of each position of the cylinder head to the mechanical properties of the head plate. At room temperature, the head plate area has the best mechanical properties, the floor plate area is second, and the thick partition plate area has the worst mechanical properties. Compared with the head plate position, the tensile strength of the thick partition plate position is reduced by $15.3 \%$, the yield strength is reduced by $4.2 \%$, and the break elongation is reduced by $67.5 \%$; the tensile strength of the floor plate is reduced by $10.4 \%$, the yield strength is reduced by $1.2 \%$, and the break elongation is reduced by $59.5 \%$. There is a slight difference in yield strength and hardness, while the difference in ultimate tensile strength and elongation at break is more obvious.

Temperature is another important factor that cannot be ignored which affect the mechanical properties of materials. The increase of working temperature greatly reduces the mechanical properties of materials. The maximum temperature of the fire surface of the cylinder head floor plate can reach $300^{\circ} \mathrm{C}$, so it is very significant to study the mechanical properties of the same position at different temperatures. Fig.4 shows the ratio of the mechanical properties of the floor plate to the room temperature mechanical properties at each corresponding temperature, which directly reflects the attenuation of the mechanical properties of the material. By comparing the mechanical properties of floor plate at different temperatures, it can be seen that the tensile strength and yield strength at room temperature are $292 \mathrm{MPa}$ and $256 \mathrm{MPa}$, respectively. With the increase of temperature, the tensile strength and yield strength at $300{ }^{\circ} \mathrm{C}$ have decreased to $98 \mathrm{MPa}$ and $88 \mathrm{MPa}$, respectively, by $66.4 \%$ and $65.6 \%$, and the break elongation has increased from $2.4 \%$ to $14.5 \%$, The increase range is $505.2 \%$, the plasticity of the material is greatly improved. The hardness also decreases with the increase in temperature, from $23^{\circ} \mathrm{C}$ to $300^{\circ} \mathrm{C}$, the hardness decreases by $41.2 \%$. When the material is in the state of high temperature and constant load for a long time, not only creep behavior will occur, the microstructure, especially the morphology of eutectic silicon and intermetallic strengthening phase will change else, resulting in the degradation of mechanical properties.

Table 2. Test results of mechanical properties.

\begin{tabular}{|c|c|c|c|c|c|}
\hline Location & T/ & HBW & UTS/MPa & YS/MPa & E/\% \\
\hline Head plate & 23 & 118 & 326 & 259 & 5.93 \\
\hline Partition plate & 23 & 112 & 276 & 248 & 1.93 \\
\hline Floor plate & 23 & 114 & 292 & 256 & 2.4 \\
\hline Floor plate & 200 & 110 & 243 & 226 & 3.53 \\
\hline Floor plate & 250 & 96 & 194 & 181 & 5.5 \\
\hline Floor plate & 300 & 67 & 98 & 88 & 14.5 \\
\hline
\end{tabular}




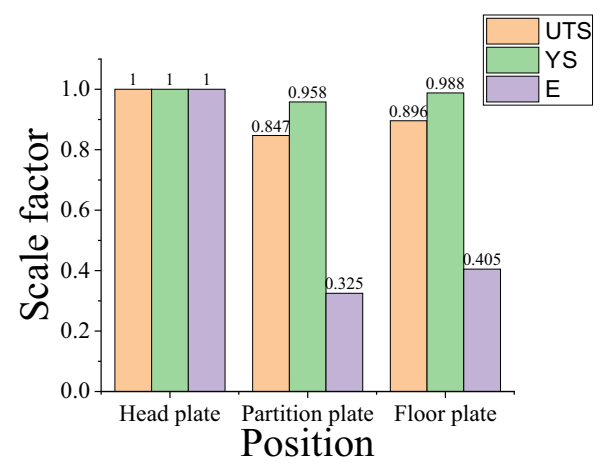

Fig. 3. Mechanical properties at different positions.

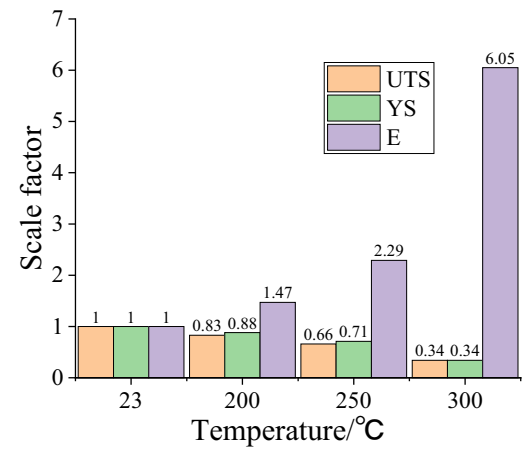

Fig. 4. Mechanical properties at different temperatures.

The partitioned mechanical properties showed that the mechanical properties of cylinder head are non-uniform distribution with space. The mechanical properties of the head plate are better than other two positions, especially in the break elongation and tensile strength. The difference of yield strength is relatively small, because the yield strength is mainly affected by the strengthening phase of intermetallic interaction produced during heat treatment. The mechanical properties of materials gradually decay as the temperature increases. From $23^{\circ} \mathrm{C}$ to $300^{\circ} \mathrm{C}$, the yield strength and tensile strength decrease in the same extent, the break elongation increases greatly, and the plasticity of the material is greatly improved.

\subsection{Microstructure}

The material of cylinder head is typical hypoeutectic Al -Si alloy, which consists of primary aluminum dendrites, eutectic silicon, intermetallic compounds and casting defects, such as shrinkage porosity, gas, oxides. The metallographic samples at three different positions of the cylinder heads are observed under optical microscope as shown in Fig.5 A-1 to C-1. The results showed that the aluminum-based cell is separated by eutectic silicon particles, and there are a small amount of intermetallic compounds $\beta$ Phase and casting defects.

The SDAS of aluminum alloy is mainly affected by the cooling rate of liquid aluminum. Some studies have shown that $\mathrm{SDAS}=\mathrm{A} \times \mathrm{R}^{\mathrm{B}}$, where $\mathrm{A}$ and $\mathrm{B}$ are constants respectively, $\mathrm{R}=\mathrm{DT} / \mathrm{Dt}$ represents the cooling rate of a local area during the casting process ${ }^{15}$. The greater the cooling rate, the higher the nucleation temperature, and the better the degree of subcooling during crystallization, results in higher 
The dendrite nucleation rate. Because there is not enough time to complete the growth and aggregation process, the distance between the secondary dendrite arms is smaller.

Fig.5 A-2 to C-2 shows the grain morphology of three different sampling positions. The grain sizes are obviously different, the head plate area is the smallest, followed by the floor plate area, and the grain size of the partition plate area is the largest. Due to the addition of nucleating agent during the casting process, the grain size distribution is relatively uniform for the same location. The grain size of the head plate area is $306 \mu \mathrm{m}$, the partition plate area is $578 \mu \mathrm{m}$, and the floor plate area is $345 \mu \mathrm{m}$. Grain refinement increases the number of grain boundaries, which can act as obstacles to the slip of dislocations and slow down the transfer and accumulation of dislocations. The role of grain boundaries hindering the slip is conducive to the plastic deformation of materials, and finally improves the elongation and tensile strength of materials. At the same time, due to the arrangement of chill in the head plate area, the cooling rate is the fastest. The partition plate area has poor heat dissipation result in the lowest cooling rate. The SDAS of head plate, partition plate and floor plate are $24 \mu \mathrm{m}, 28 \mu \mathrm{m}$ and $56 \mu \mathrm{m}$, respectively. The decrease of SDAS will also increase the grain boundary and improve the mechanical properties of the material.

Fig.5 A-3 to C-3 shows the comparison of eutectic silicon morphology at different sampling positions. The morphology of eutectic silicon in the head and floor area is mostly short rod and oval, while the partition plate area with slow cooling rate is mostly long and narrow needle. The narrow and long silicon needles are prone to stress concentration and become the source of crack initiation, which will greatly weaken the mechanical properties of the corresponding position. In the casting process of cylinder head, the cooling rate of the head plate is the fastest, results in largest solubility and higher uniformity of supersaturated solid solution. After aging treatment, the eutectic silicon has the smallest morphology and more rounded, the dispersion strength is also higher. Table 3 shows the quantitative result of the microstructure at different positions of the cylinder head.

There are obvious differences in the microstructure of the samples at different positions of the cylinder head. From a trend point of view, the change trend of grain size, SDAS and eutectic silicon aspect ratio are the same, and there is a positive correlation among them. The smaller the grain size and SDAS are, the smaller the eutectic silicon aspect ratio is. From the position point of view, the microstructure characterization parameters of the head plate area are the best, followed by the floor plate area, and the partition plate area is the worst.

The results of the quantification of the microstructure and the tensile mechanical properties of the cylinder head showed that both of them are non-uniformly distributed with the structure of the casting. The microstructure and mechanical properties of different positions are not the same. For cylinder heads with in-situ sampling, the optimal position of the microstructure has the best tensile mechanical properties, and the worst position corresponds to the worst tensile mechanical properties. There is a strong correlation between the microstructure and the mechanical properties. It is very important to establish the relationship between microstructure and mechanical properties.

Table 3. Quantitative microstructure results.

\begin{tabular}{|c|c|c|c|}
\hline Location & Grain size/ $\boldsymbol{\mu m}$ & SDAS/ $\boldsymbol{\mu m}$ & Aspect ratio \\
\hline Head plate & 306 & $24.0 \pm 2$ & 1.47 \\
\hline Partition plate & 578 & $56.0 \pm 2$ & 3.48 \\
\hline Floor plate & 345 & $28.0 \pm 5$ & 2.05 \\
\hline
\end{tabular}

\subsection{Hall-Petch relationship}

Grain size and SDAS play a dominant role in controlling the mechanical properties of materials. Hall and Petch found that there is a quantitative relationship between the strength 
of metallic materials and the grain size according to the dislocation stacking theory of single crystal and polycrystalline materials, and this relationship is characterized by the Hall-Petch formula:

It can also be expressed by hardness as

$$
\sigma_{s}=\sigma_{0}+k d^{-\frac{1}{2}}
$$

$$
H_{v}=H_{0}+k d^{-\frac{1}{2}}
$$

\subsubsection{Hall-Petch relationship between grain size and ultimate tensile strength}

Fig.6 shows the Hall-Petch relationship between grain size and ultimate tensile strength of in-situ sample of aluminum alloy cylinder head. UTS $=96.98+4055.22 / \sqrt{d}$ the
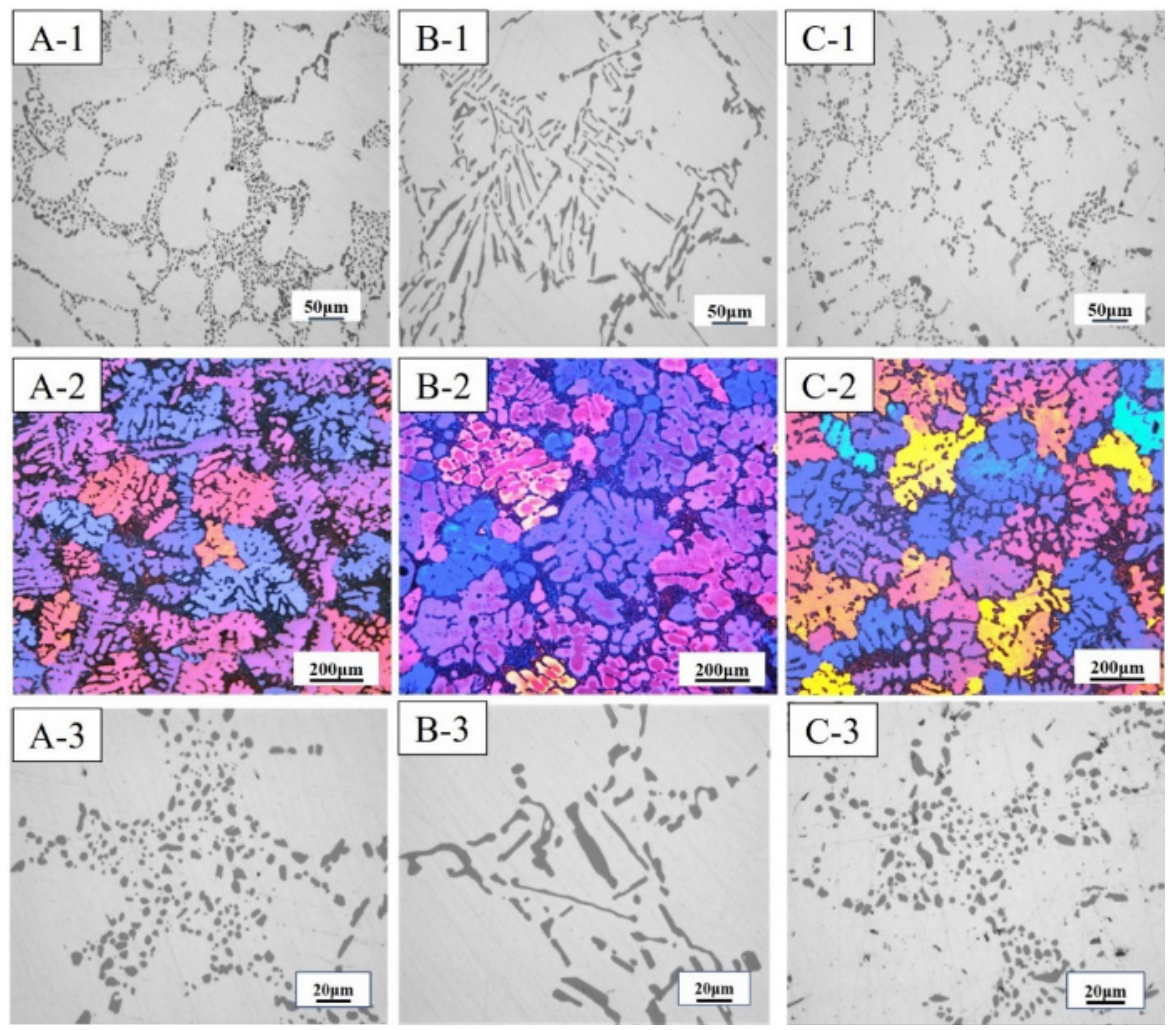

Fig. 5. Microstructure properties at different positions.

Goodness of fit $\mathrm{R}^{2}=0.877$, and the average error is $2.77 \%$. Because the free energy of the grain boundary is higher than that of the unit cell, the grain refinement makes the grain boundary increase in the material, and the grain boundary can be used as a barrier to dislocation slip and finally enhance the strength of the material. Assuming that the lower limit of the allowable tensile strength of the cylinder head material is $255 \mathrm{MPa}$, the upper limit of the allowable grain size can be obtained as:

$$
d_{\text {MAX }}=\left(\frac{4055.22}{\mathrm{UTS}_{\min }-96.98}\right)^{2}=658.6 \mu \mathrm{m}
$$

If the error limit of the Hall-Petch relationship is considered, the upper threshold of allowable grain size is: 


$$
d_{\text {MAX }}=\left(\frac{4055.22}{\mathrm{UTS} \times(1+2.77 \%)-96.98}\right)^{2}=603.4 \mu \mathrm{m}
$$

\subsubsection{Hall-Petch relationship between SDAS and ultimate tensile strength}

The SDAS and ultimate tensile strength also satisfy the Hall-Petch relationship UTS = $172.87+748.60 / \sqrt{S D A S}$, the goodness of fit $\mathrm{R}^{2}=0.850$, and the average error is $3.1 \%$. The smaller the SDAS, the finer the grains, and the grain refinement improves material strength and elongation. Similarly, assuming that the lower limit of the allowable ultimate tensile strength of the cylinder head material is $255 \mathrm{MPa}$, the upper limit of the allowable SDAS can be obtained as:

$$
S D A S_{M A X}=\left(\frac{748.6}{U T S_{\min }-172.87}\right)^{2}=83.1 \mu \mathrm{m}
$$

If the error limit of the Hall-Petch relationship is considered, the upper threshold of allowable SDAS is:

$$
S D A S_{\text {MAX }}=\left(\frac{348.6}{U_{T S S_{\text {min }} \times(1+3.1 \%)-172.87}}\right)^{2}=69.1 \mu \mathrm{m}
$$

Fig. 6. Hall-Petch relationship between grain size and ultimate tensile strength.

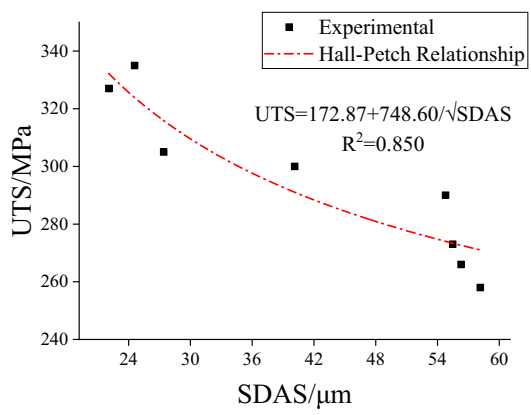

Fig. 7. Hall-Petch relationship between SDAS and ultimate tensile strength.

\subsection{The relationship between yield strength and hardness}

Hardness is simpler and easier to measure than yield strength and other mechanical properties, it is of practical significance to predict the yield strength of materials with hardness. The hardness and yield strength satisfy the nonlinear relationship $\sigma 0.2=781.0$ 5709.8/ HBW.Fig.8 shows the nonlinear relationship between hardness and yield strength. 
The error of most data is within the 5\% error limit, and the maximum error is $13.8 \%$, the average error is $4.35 \%$. The prediction error is enough to meet the engineering needs.

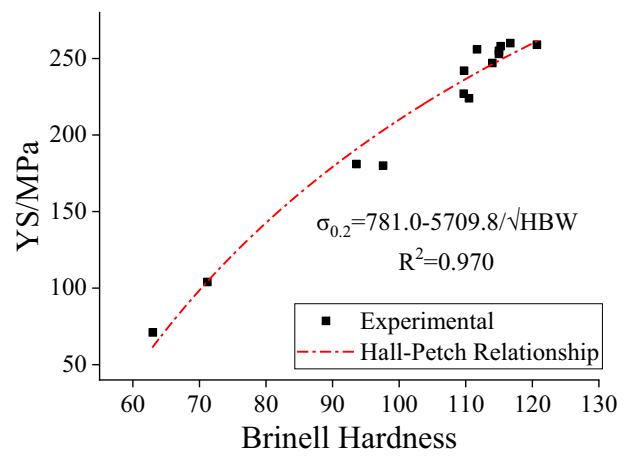

Fig. 8. Nonlinear relationship between hardness and yield strength.

\section{Conclusions}

The in-situ partitioned properties of cast aluminum alloy cylinder head have been studied experimentally. The following conclusions can be drawn.

1) The in-situ mechanical properties and microstructure of cylinder head are non-uniform distribution. The mechanical properties and microstructure of the head plate are the best, followed by the floor plate and the partition plate, especially in ultimate tensile strength and break elongation. The mechanical properties of the floor plate decrease with the increase of temperature, from $23^{\circ} \mathrm{C}$ to $300^{\circ} \mathrm{C}$, the tensile strength and yield strength decrease in the same range, but the break elongation changes most obviously.

2) There is an obvious Hall-Petch relationship between the microstructure and mechanical properties of the cylinder head. If the required ultimate tensile strength is not less than $255 \mathrm{MPa}$, the upper threshold of the grain size, by considering the error limit of the Hall-Petch relationship, is $603.4 \mu \mathrm{m}$, and the SDAS is $69.1 \mu \mathrm{m}$.

3) The hardness and yield strength of the cylinder head meet the nonlinear relationship $Y S=781.0-5709.8 / \sqrt{H B W}$.

\section{References}

1. M. J. WANG, D. Y. HUANG and H. T. JIANG, Heat Treatment of Metals (2006)

2. L. ZHEN, W. D. FEI, S. B. KANG and H. W. KIM, J. Mater. Sci. 32(1997)

3. B. Baradarani and R. Raiszadeh, Mater. Design, 32, (2011)

4. L. Ceschini, A. Morri and A. Morri, J. Mater. Eng. Perform. 22 (2013)

5. X. Zhang, H. Yu and C. Li, J. Mater. Process. Tech. 227 (2016)

6. J. Li, D. Nong, K. Zheng, X. Li and M. Zhao, J. Cent South Univ, 21(2014).

7. X. SHE, X. JIANG, P. WANG, B. TANG, K. CHEN, Y. LIU and W. CAO, T. Nonferr. Metal. Soc. 30(2020)

8. M. Okayasu, Y. Ohkura, S. Takeuchi, S. Takasu, H. Ohfuji and T. Shiraishi, Materials Science and Engineering: A, 543(2012)

9. T. Liu, Y. Li and Y. Ren, Mater. Lett. 214(2017) 
10. E. Ghassemali, M. Riestra, T. Bogdanoff, B. S. Kumar and S. Seifeddine, Procedia Engineering, 207 (2017)

11. M. Zamani, S. Seifeddine and A. E. W. Jarfors, Mater. Design, 86 (2015)

12. W. Li, S. Li, J. Liu, A. Zhang, Y. Zhou, Q. Wei, C. Yan and Y. Shi, Materials Science and Engineering: A, 663 (2016)

13. L. Wenyi, X. Cong, L. Maowen, X. Wenlong and M. Chaoli, Journal of Materials Engineering, 47 (2019)

14. S. Hegde and K. N. Prabhu, J. Mater. Sci. 43 (2008)

15. Q. G. Wang, Metallurgical and materials transactions. A, Physical metallurgy and materials science, 34 (2003) 